\title{
Improved Lower Bounds on the Area Requirements of Series-Parallel Graphs ${ }^{\star}$
}

\author{
Fabrizio Frati \\ Dipartimento di Informatica e Automazione, Università Roma Tre \\ frati@dia.uniroma3.it
}

\begin{abstract}
We show that there exist series-parallel graphs requiring $\Omega\left(n 2^{\sqrt{\log n}}\right)$ area in any straight-line or poly-line grid drawing, improving the previously best known $\Omega(n \log n)$ lower bound.
\end{abstract}

\section{Introduction}

Determining asymptotic bounds for the area requirements of straight-line and poly-line drawings of planar graphs is a classical topic in the Graph Drawing literature. Groundbreaking works of the beginning of the nineties [5]10] have shown that every $n$-vertex planar graph admits a planar straight-line drawing in an $O\left(n^{2}\right)$ grid. Such a bound is worst-case optimal, even for poly-line drawings [7|5]. Hence, it is natural to search for interesting sub-classes of planar graphs admitting sub-quadratic area drawings.

In this paper we deal with series-parallel graphs, a class of planar graphs that has been widely investigated in Graph Theory and Graph Drawing (see, e.g., [118 16]). Series-parallel graphs can be equivalently defined as the graphs excluding $K_{4}$ as a minor or, inductively, by series and parallel compositions of smaller series-parallel graphs.

Biedl [2] proved that a series-parallel graph with $n$ vertices admits a poly-line grid drawing in $O\left(n^{3 / 2}\right)$ area. She achieved such a bound by first constructing visibility representations of series-parallel graphs in $O\left(n^{3 / 2}\right)$ area and by then turning such representations into poly-line drawings with asymptotically the same area. No sub-quadratic area upper bound is known for straight-line grid drawings of series-parallel graphs.

The author proved [9] that there exist series-parallel graphs requiring $\Omega(n \log n)$ area in any straight-line or poly-line grid drawing. To achieve such a bound, the following theorem 1 was proved in [9], improving upon previous results of Biedl et al. [4].

Theorem 1. Every planar straight-line or poly-line grid drawing of $K_{2, n}$ in a $W \times H$ grid satisfies $\max \{W, H\} \geq c \cdot n$, for some constant $c \leq 1 / 2$.

In this paper, we prove the following.

Theorem 2. There exist series-parallel graphs with $n$ vertices requiring $\Omega\left(2^{\sqrt{\log n}}\right)$ width and $\Omega\left(2^{\sqrt{\log n}}\right)$ height in any straight-line or poly-line grid drawing.

\footnotetext{
* This work is partially supported by the Italian Ministry of Research, Grant number RBIP06BZW8, FIRB project "Advanced tracking system in intermodal freight transportation".

${ }^{1}$ Theorem 1 is stated in [9] in an equivalent form using the $\Omega(n)$ notation. 
Such a result is achieved by carefully constructing a graph out of several copies of $K_{2, n}$ and by then exploiting Theorem 1 and some further geometric considerations. Theorem 1, together with Theorem 2] directly implies the following.

Theorem 3. There exist series-parallel graphs with $n$ vertices requiring $\Omega\left(n 2^{\sqrt{\log n}}\right)$ area in any straight-line or poly-line grid drawing.

We remark that the function $2^{\sqrt{\log n}}$ is asymptotically greater than any polylogarithmic function of $n$ and smaller than any polynomial function of $n$.

\section{Preliminaries}

A planar grid drawing of a graph is a mapping of each vertex to a distinct point of the plane with integer coordinates and of each edge to a Jordan curve between the endpoints of the edge, so that no two edges intersect except, possibly, at common endpoints. In the following we always refer to planar grid drawings. A straight-line drawing is such that all edges are rectilinear segments. A poly-line drawing is such that the edges are sequences of rectilinear segments. In a poly-line drawing a bend is a point in which an edge changes its slope, i.e., a point common to two consecutive segments in the sequence of segments representing the edge. In a grid drawing bends have integer coordinates. A polygonal path is a poly-line grid drawing of a path. The bounding box of a drawing $\Gamma$ is the smallest rectangle with sides parallel to the axes that covers $\Gamma$ completely. The height (width) of $\Gamma$ is the height (resp. width) of its bounding box. The area of $\Gamma$ is the height of $\Gamma$ times its width.

A drawing of the complete bipartite graph $K_{2, n}$ can be thought as a drawing of $n$ paths that start and end at the same two vertices and that do not share any other vertex. In the following we will refer to such paths as to the paths of $K_{2, n}$.

In the next section, we will use the following lemmata [9].

Lemma 1. Consider any poly-line grid drawing of $K_{2, n}$, any path $\pi$ of $K_{2, n}$, and any vector $\boldsymbol{v}$. There exists a grid point $p \in \pi$ such that $\boldsymbol{v} \cdot p \geq \boldsymbol{v} \cdot p^{\prime}$, for any point $p^{\prime} \in \pi$.

Lemma 2. Let $a$ and $b$ be the endvertices of the paths of $K_{2, n}$. Consider any planar drawing of $K_{2, n}$. Let l be any line that does not intersect nor contain the open segment $\overline{a b}$. No three paths $\pi_{1}, \pi_{2}$, and $\pi_{3}$ of $K_{2, n}$ exist such that: (i) $\pi_{1}, \pi_{2}$, and $\pi_{3}$ do not intersect each other; (ii) $\pi_{1}, \pi_{2}$, and $\pi_{3}$ are contained in the closed half-plane delimited by $l$ and containing $a$ and $b$; (iii) each of $\pi_{1}, \pi_{2}$, and $\pi_{3}$ touches $l$ at least once.

\section{Proof of Theorem 2}

As straight-line drawings are also poly-line drawings, it suffices to prove Theorem2 for poly-line drawings. Let $f(n)$ be a function to be computed later and let $d=c / 4$, where $c$ is the constant of Theorem 1 Observe that $d \leq 1 / 8$.

Graph $G_{1}$ is $K_{2, f(n)-2}$. Graph $G_{i+1}$ is defined as follows. Consider $f(n)$ copies $G_{i, 1,1}, G_{i, 1,2}, G_{i, 2,1}, G_{i, 2,2}, \ldots, G_{i, j, 1}, G_{i, j, 2}, \ldots, G_{i, f(n) / 2,1}, G_{i, f(n) / 2,2}$ of $G_{i}$; construct $f(n) / 2$ series-parallel graphs $G_{i, 1}, G_{i, 2}, \ldots, G_{i, j}, \ldots, G_{i, f(n) / 2}$, where $G_{i, j}$ is 


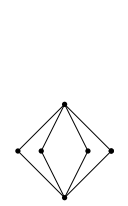

(a)

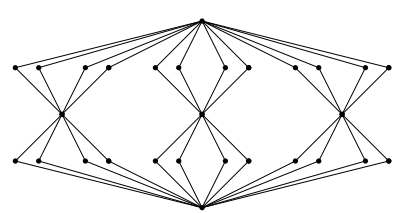

(b)

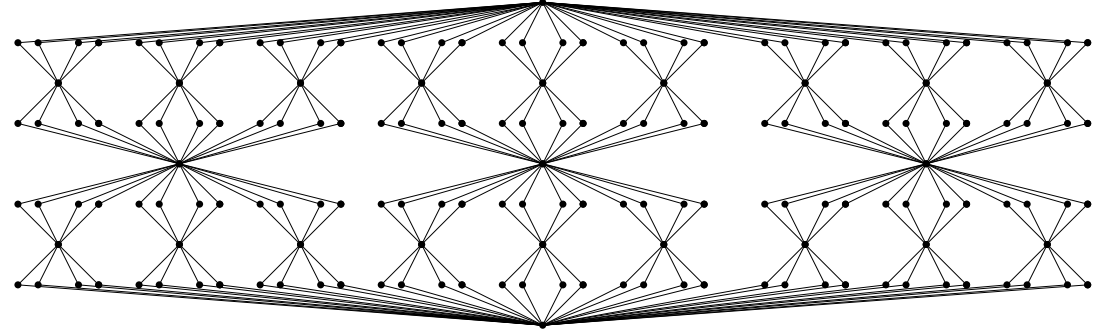

(c)

Fig. 1. Graphs $G_{i}$, with $f(n)=6$. (a) $G_{1}$. (b) $G_{2}$. (c) $G_{3}$

the series composition of $G_{i, j, 1}$ and $G_{i, j, 2}$; then, $G_{i+1}$ is the parallel composition of graphs $G_{i, 1}, G_{i, 2}, \ldots, G_{i, f(n) / 2}$. See Fig. 1.

First, we prove Theorem 2 for sufficiently large graphs, that is, for graphs having a number of vertices that is at least some constant $n_{0}$ to be determined later. From now till it is otherwise specified, assume that $n \geq n_{0}$.

Suppose that $f(n) \geq 8, \forall n \geq n_{0}$. Let $n$ be the number of vertices of graph $G_{k}$. We have the following main lemma.

Lemma 3. Let $\Gamma_{i}$ be any poly-line grid drawing of $G_{i}$ and let $a_{i}$ and $b_{i}$ be the poles of $G_{i}$, for each $1 \leq i \leq k$. Then, one of the following holds:

- Condition 1: The height and the width of $\Gamma_{i}$ are both greater than or equal to $d \cdot f(n)$.

- Condition 2: The width of $\Gamma_{i}$ is greater than or equal to $d \cdot f(n)$ and $\Gamma_{i}$ contains a polygonal path $l_{i}$ connecting $a_{i}$ to $b_{i}$ that has height greater than or equal to $2^{i}$ and such that, for every point $p \in l_{i}, \min \left\{y\left(a_{i}\right), y\left(b_{i}\right)\right\} \leq y(p) \leq \max \left\{y\left(a_{i}\right), y\left(b_{i}\right)\right\}$; or the height of $\Gamma_{i}$ is greater than or equal to $d \cdot f(n)$ and $\Gamma_{i}$ contains a polygonal path $l_{i}$ connecting $a_{i}$ to $b_{i}$ that has width greater than or equal to $2^{i}$ and such that, for every point $p \in l_{i}, \min \left\{x\left(a_{i}\right), x\left(b_{i}\right)\right\} \leq x(p) \leq \max \left\{x\left(a_{i}\right), x\left(b_{i}\right)\right\}$.

Proof: We prove the statement by induction on $i$. In the base case, consider any polyline grid drawing $\Gamma_{1}$ of $G_{1}$. By Theorem 1 one of the height and the width of $\Gamma_{1}$, say the width of $\Gamma_{1}$, is at least $c \cdot f(n)$, hence it is at least $d \cdot f(n)$.

Assume, without loss of generality, that $y\left(a_{1}\right) \leq y\left(b_{1}\right)$. Suppose that at least $2 d \cdot f(n)$ paths of $G_{1}=K_{2, f(n)-2}$ intersect the open half-plane $H^{-}\left(a_{1}\right)$ defined as $y<y\left(a_{1}\right)$ or the open half-plane $H^{+}\left(b_{1}\right)$ defined as $y>y\left(b_{1}\right)$. By Lemma 1 with $\boldsymbol{v}=(0,-1)$, for each path $\pi$ of $G_{1}$ intersecting $H^{-}\left(a_{1}\right)$, a grid point $p \in \pi$ exists whose $y$-coordinate 
is minimum among the points of $\pi$. Clearly, $p$ belongs to $H^{-}\left(a_{1}\right)$. Hence, $p$ belongs to a horizontal grid line $h$ not intersecting nor containing segment $\overline{a_{1} b_{1}}$. By Lemma 2 , at most two paths of $G_{1}$ have their points with smallest $y$-coordinate in $h$. Analogously, by Lemma 1 with $\boldsymbol{v}=(0,1)$, for each path $\pi$ of $G_{1}$ that intersects $H^{+}\left(b_{1}\right)$, a grid point $p \in \pi$ exists whose $y$-coordinate is maximum among the points of $\pi$. Clearly, $p$ belongs to $H^{+}\left(b_{1}\right)$. Hence, $p$ belongs to a horizontal grid line $h$ not intersecting nor containing segment $\overline{a_{1} b_{1}}$. By Lemma2 at most two paths of $G_{1}$ have their points with greatest $y$ coordinate in $h$. Hence, as $2 d \cdot f(n)$ paths of $G_{1}$ intersect $H^{-}\left(a_{1}\right)$ or $H^{+}\left(b_{1}\right)$, it follows that $\Gamma_{1}$ has height at least $d \cdot f(n)$.

Now suppose that no $2 d \cdot f(n)$ paths of $G_{1}$ intersect $H^{-}\left(a_{1}\right)$ or $H^{+}\left(b_{1}\right)$. Then, since $d \leq 1 / 8$, at least $f(n)-2-2 d \cdot f(n)+1 \geq 3 f(n) / 4-1$ paths of $G_{1}$ are such that, for every point $p$ of any such a path, $y\left(a_{1}\right) \leq y(p) \leq y\left(b_{1}\right)$. By planarity of $\Gamma_{1}$ at most one path of $G_{1}$ contains a point $p$ such that $y(p)=y\left(a_{1}\right)$ and $x(p)<x\left(a_{1}\right)$. Analogously, at most one path of $G_{1}$ contains a point $p$ such that $y(p)=y\left(a_{1}\right)$ and $x(p)>x\left(a_{1}\right)$, at most one path of $G_{1}$ contains a point $p$ such that $y(p)=y\left(b_{1}\right)$ and $x(p)<x\left(b_{1}\right)$, and at most one path of $G_{1}$ contains a point $p$ such that $y(p)=y\left(b_{1}\right)$ and $x(p)>x\left(b_{1}\right)$. Since $f(n) \geq 8$, it follows that $3 f(n) / 4-1 \geq 5$, hence there is at least one path of $G_{1}$ whose only vertex $v \neq a_{1}, b_{1}$ is such that $y(v)>y\left(a_{1}\right)$ and $y(v)<y\left(b_{1}\right)$. Then, the polygonal path $\left(a_{1}, v, b_{1}\right)$ has height at least two and is such that, for every point $p \in\left(a_{1}, v, b_{1}\right), y\left(a_{1}\right) \leq y(p) \leq y\left(b_{1}\right)$, thus proving the base case.

In the inductive case, consider any poly-line grid drawing $\Gamma_{i+1}$ of $G_{i+1}$, containing drawings $\Gamma_{i, 1,1}, \Gamma_{i, 1,2}, \Gamma_{i, 2,1}, \Gamma_{i, 2,2}, \ldots, \Gamma_{i, j, 1}, \Gamma_{i, j, 2}, \ldots, \Gamma_{i, f(n) / 2,1}, \Gamma_{i, f(n) / 2,2} \quad$ of $G_{i, 1,1}, G_{i, 1,2}, G_{i, 2,1}, G_{i, 2,2}, \ldots, G_{i, j, 1}, G_{i, j, 2}, \ldots, G_{i, f(n) / 2,1}, G_{i, f(n) / 2,2}$, respectively. By induction, for $1 \leq j \leq f(n) / 2$ and $1 \leq k \leq 2, \Gamma_{i, j, k}$ satisfies Condition 1 or 2 .

If two indices $1 \leq j \leq f(n) / 2$ and $1 \leq k \leq 2$ exist such that $\Gamma_{i, j, k}$ satisfies Condition 1, then the width and the height of $\Gamma_{i, j, k}$ are both greater than or equal to $d \cdot f(n)$, hence so are the width and the height of $\Gamma_{i+1}$.

Hence, we can assume that, for every $1 \leq j \leq f(n) / 2$ and $1 \leq k \leq 2, \Gamma_{i, j, k}$ satisfies Condition 2. If indices $1 \leq j^{\prime}, j^{\prime \prime} \leq f(n) / 2$ and $1 \leq k^{\prime}, k^{\prime \prime} \leq 2$ exist, where $j^{\prime}=j^{\prime \prime}$ and $k^{\prime}=k^{\prime \prime}$ do not hold simultaneously, such that the width of $\Gamma_{i, j^{\prime}, k^{\prime}}$ is greater than or equal to $d \cdot f(n)$ and the height of $\Gamma_{i, j^{\prime \prime}, k^{\prime \prime}}$ is greater than or equal to $d \cdot f(n)$, then the width and the height of $\Gamma_{i+1}$ are both greater than or equal to $d \cdot f(n)$.

Hence, we can assume that, for every $1 \leq j \leq f(n) / 2$ and $1 \leq k \leq 2$, the width of $\Gamma_{i, j, k}$ is greater than or equal to $d \cdot f(n)$ and $\Gamma_{i, j, k}$ contains a polygonal path $l_{i, j, k}$ connecting $a_{i}$ to $b_{i}$ that has height greater than or equal to $2^{i}$ and such that, for every point $p \in l_{i, j, k}, \min \left\{y\left(a_{i}\right), y\left(b_{i}\right)\right\} \leq y(p) \leq \max \left\{y\left(a_{i}\right), y\left(b_{i}\right)\right\}$; the case in which, for every $1 \leq j \leq f(n) / 2$ and $1 \leq k \leq 2$, the height of $\Gamma_{i, j, k}$ is greater than or equal to $d \cdot f(n)$ and $\Gamma_{i, j, k}$ contains a polygonal path $l_{i, j, k}$ connecting $a_{i}$ to $b_{i}$ that has width greater than or equal to $2^{i}$ and such that, for every point $p \in l_{i, j, k}, \min \left\{x\left(a_{i}\right), x\left(b_{i}\right)\right\} \leq$ $x(p) \leq \max \left\{x\left(a_{i}\right), x\left(b_{i}\right)\right\}$ can be treated analogously.

Denote by $l_{i, j}$ the path connecting $a_{i+1}$ and $b_{i+1}$ composed of $l_{i, j, 1}$ and $l_{i, j, 2}$. Assume, without loss of generality, that $y\left(a_{i+1}\right) \leq y\left(b_{i+1}\right)$. Suppose that at least $2 d \cdot f(n)$ paths $l_{i, j}$ intersect the open half-plane $H^{-}\left(a_{i+1}\right)$ defined as $y<y\left(a_{i+1}\right)$ or the open half-plane $H^{+}\left(b_{i+1}\right)$ defined as $y>y\left(b_{i+1}\right)$. By Lemma 1 with $\boldsymbol{v}=(0,-1)$, for each path $l_{i, j}$ that intersects $H^{-}\left(a_{i+1}\right)$, a grid point $p \in l_{i, j}$ exists whose $y$-coordinate 
is minimum among the points of $l_{i, j}$. Clearly, $p$ belongs to $H^{-}\left(a_{i+1}\right)$. Hence, $p$ belongs to a horizontal grid line $h$ not intersecting nor containing segment $\overline{a_{i+1} b_{i+1}}$. By Lemma2, at most two paths $l_{i, j}$ have their points with smallest $y$-coordinate in $h$. Analogously, by Lemma 1 with $\boldsymbol{v}=(0,1)$, for each path $l_{i, j}$ that intersects $H^{+}\left(b_{i+1}\right)$, a grid point $p \in l_{i, j}$ exists whose $y$-coordinate is maximum among the points of $l_{i, j}$. Clearly, $p$ belongs to $H^{+}\left(b_{i+1}\right)$. Hence, $p$ belongs to a horizontal grid line $h$ not intersecting nor contain segment $\overline{a_{i+1} b_{i+1}}$. By Lemma 2] at most two paths $l_{i, j}$ have their points with greatest $y$-coordinate in $h$. Hence, as $2 d \cdot f(n)$ paths $l_{i, j}$ intersect $H^{-}\left(a_{i+1}\right)$ or $H^{+}\left(b_{i+1}\right)$, it follows that $\Gamma_{i+1}$ has height at least $d \cdot f(n)$.

Now suppose that no $2 d \cdot f(n)$ paths $l_{i, j}$ intersect $H^{-}\left(a_{i+1}\right)$ or $H^{+}\left(b_{i+1}\right)$. Then, since $d \leq 1 / 8$, at least $f(n)-2 d \cdot f(n)+1 \geq 3 f(n) / 4+1$ paths $l_{i, j}$ are such that, for every point $p$ of any such a path, $y\left(a_{i+1}\right) \leq y(p) \leq y\left(b_{i+1}\right)$. By planarity of $\Gamma_{i+1}$ at most one path $l_{i, j}$ contains a point $p$ such that $y(p)=y\left(a_{i+1}\right)$ and $x(p)<x\left(a_{i+1}\right)$. Analogously, at most one path $l_{i, j}$ contains a point $p$ such that $y(p)=y\left(a_{i+1}\right)$ and $x(p)>x\left(a_{i+1}\right)$, at most one path $l_{i, j}$ contains a point $p$ such that $y(p)=y\left(b_{i+1}\right)$ and $x(p)<x\left(b_{i+1}\right)$, and at most one path $l_{i, j}$ contains a point $p$ such that $y(p)=y\left(b_{i+1}\right)$ and $x(p)>x\left(b_{i+1}\right)$. Since $f(n) \geq 8$, it follows that $3 f(n) / 4+1 \geq 5$, hence there is at least one path $l_{i, j}$ composed of path $l_{i, j, 1}$, that connects the poles $a_{i+1}$ and $v$ of $G_{i, j, 1}$, and of path $l_{i, j, 2}$, that connects the poles $b_{i+1}$ and $v$ of $G_{i, j, 2}$, such that $y(v)>y\left(a_{i+1}\right)$ and $y(v)<y\left(b_{i+1}\right)$. By inductive hypothesis, $l_{i, j, 1}$ has height greater than or equal to $2^{i}$ and, for every point $p \in l_{i, j, 1}, y\left(a_{i+1}\right) \leq y(p) \leq y(v)$; further, $l_{i, j, 2}$ has height greater than or equal to $2^{i}$ and, for every point $p \in l_{i, j, 2}, y(v) \leq y(p) \leq y\left(b_{i+1}\right)$; hence, $l_{i, j}$ has height greater than or equal to $2^{i+1}$ and, for every point $p \in l_{i, j}, y\left(a_{i+1}\right) \leq y(p) \leq$ $y\left(b_{i+1}\right)$, thus completing the induction.

Corollary 1. Any poly-line grid drawing of $G_{k}$ has height and width that are both greater than or equal to $\min \left\{d \cdot f(n), 2^{k}\right\}$.

Let $f(n)=n^{x(k)}$. By construction $\left|G_{1}\right|=n^{x(k)}$; since $G_{i}$ is composed of $f(n)=$ $n^{x(k)}$ copies of $G_{i-1},\left|G_{i}\right| \leq n^{x(k)} \cdot\left|G_{i-1}\right|$; inductively, we obtain $\left|G_{k}\right| \leq n^{k \cdot x(k)}$. Assuming $\left|G_{k}\right|=n$, we get $x(k) \geq 1 / k$ and $f(n) \geq n^{1 / k}$.

We now choose $k$ in such a way that $n^{1 / k}$ and $2^{k}$ are equal. This is done as follows. $2^{k}=n^{1 / k} \Rightarrow \log _{2}\left(2^{k}\right)=\log _{2}\left(n^{1 / k}\right) \Rightarrow k \log _{2}(2)=1 / k \log _{2}(n) \Rightarrow k^{2}=$ $\log _{2}(n) \Rightarrow k=\sqrt{\log _{2}(n)}$. By Corollary 11 both the height and the width of $\Gamma_{k}$, with $k=\sqrt{\log _{2}(n)}$, are greater than or equal to $\min \left\{d \cdot n^{1 / \sqrt{\log _{2}(n)}}, 2^{\sqrt{\log _{2}(n)}}\right\}=$ $d \cdot 2^{\sqrt{\log _{2}(n)}}=\Omega\left(2^{\sqrt{\log _{2}(n)}}\right)$, and Theorem 2 follows if $n \geq n_{0}$.

As we need $f(n)=2^{\sqrt{\log _{2}(n)}} \geq 8, \forall n \geq n_{0}$, then $n_{0}=512$. However, $d \cdot 2^{\sqrt{\log _{2}(n)}}<$ 1 , for all $n<512$, as $d \leq 1 / 8$. Since every drawing of a graph that is not a collection of paths has height and width at least one, the $d \cdot 2^{\sqrt{\log _{2}(n)}}$ lower bound holds also for graphs with less than 512 nodes, thus completing the proof of Theorem 2 .

\section{Conclusions and Open Problems}

In this paper we have shown that there exist series-parallel graphs with $n$ vertices requiring $\Omega\left(n 2^{\sqrt{\log n}}\right)$ area in any straight-line or poly-line grid drawing. 
The best known area upper bound for poly-line grid drawings of series-parallel graphs is $O\left(n^{3 / 2}\right)$ [2]3], while no sub-quadratic area upper bound is known in the case of straight-line grid drawings. Hence, in both cases, closing the gap between upper and lower bound is an intriguing challenge.

Concerning straight-line drawings, David Wood [12] conjectures the following: Let $p_{1}, \ldots, p_{k}$ be positive integers. Let $G\left(p_{1}\right)$ be the graph obtained from $K_{3}$ by adding $p_{1}$ new vertices adjacent to $v$ and $w$ for each edge $(v, w)$ of $K_{3}$. For $k \geq 2$, let $G\left(p_{1}, p_{2}, \ldots, p_{k}\right)$ be the graph obtained from $G\left(p_{1}, p_{2}, \ldots, p_{k-1}\right)$ by adding $p_{k}$ new vertices adjacent to $v$ and $w$ for each edge $(v, w)$ of $G\left(p_{1}, p_{2}, \ldots, p_{k-1}\right)$. Observe that $G\left(p_{1}, p_{2}, \ldots, p_{k}\right)$ is a series-parallel graph.

Conjecture 1. (D. R. Wood) Every straight-line grid drawing of $G\left(p_{1}, p_{2}, \ldots, p_{k}\right)$ requires $\Omega\left(n^{2}\right)$ area for some choice of $k$ and $p_{1}, p_{2}, \ldots, p_{k}$.

\section{Acknowledgments}

Thanks to Patrizio Angelini and Giuseppe Di Battista for very useful discussions. Also thanks to David Wood for sharing his strong conjecture.

\section{References}

1. Bertolazzi, P., Cohen, R.F., di Battista, G., Tamassia, R., Tollis, I.G.: How to draw a seriesparallel digraph. International Journal of Computational Geometry \& Applications 4(4), 385-402 (1994)

2. Biedl, T.C.: Small poly-line drawings of series-parallel graphs. Tech. Report CS-2007-23, School of Computer Science, University of Waterloo, Canada (2005)

3. Biedl, T.C.: On small drawings of series-parallel graphs and other subclasses of planar graphs. In: Eppstein, D., Gansner, E.R. (eds.) GD 2009. LNCS, vol. 5849, pp. 280-291. Springer, Heidelberg (2010)

4. Biedl, T.C., Chan, T.M., López-Ortiz, A.: Drawing $K_{2, n}$ : A lower bound. Information Processing Letters 85(6), 303-305 (2003)

5. de Fraysseix, H., Pach, J., Pollack, R.: How to draw a planar graph on a grid. Combinatorica 10(1), 41-51 (1990)

6. Di Giacomo, E., Didimo, W., Liotta, G., Wismath, S.K.: Book embeddability of seriesparallel digraphs. Algorithmica 45(4), 531-547 (2006)

7. Dolev, D., Leighton, T., Trickey, H.: Planar embeddings of planar graphs. Advances in Computing Research 2, 147-161 (1984)

8. Eppstein, D.: Parallel recognition of series-parallel graphs. Information and Computation 98(1), 41-55 (1992)

9. Frati, F.: A lower bound on the area requirements of series-parallel graphs. In: Broersma, H., Erlebach, T., Friedetzky, T., Paulusma, D. (eds.) WG 2008. LNCS, vol. 5344, pp. 159-170. Springer, Heidelberg (2008)

10. Schnyder, W.: Embedding planar graphs on the grid. In: ACM-SIAM Symposium on Discrete Algorithms (SODA 1990), pp. 138-148 (1990)

11. Valdes, J., Tarjan, R.E., Lawler, E.L.: The recognition of series parallel digraphs. SIAM Journal on Computing 11(2), 298-313 (1982)

12. Wood, D.R.: Private Communication (2008) 